\title{
A hidden analytic structure of the Rabi model
}

\author{
Alexander Moroz \\ Wave-scattering.com
}

\begin{abstract}
The Rabi model describes the simplest interaction between a cavity mode with a frequency $\omega_{c}$ and a two-level system with a resonance frequency $\omega_{0}$. It is shown here that the spectrum of the Rabi model coincides with the support of the discrete Stieltjes integral measure in the orthogonality relations of recently introduced orthogonal polynomials. The exactly solvable limit of the Rabi model corresponding to $\Delta=\omega_{0} /\left(2 \omega_{c}\right)=0$, which describes a displaced harmonic oscillator, is characterized by the discrete Charlier polynomials in normalized energy $\epsilon$, which are orthogonal on an equidistant lattice. A non-zero value of $\Delta$ leads to non-classical discrete orthogonal polynomials $\phi_{k}(\epsilon)$ and induces a deformation of the underlying equidistant lattice. The results provide a basis for a novel analytic method of solving the Rabi model. The number of ca. 1350 calculable energy levels per parity subspace obtained in double precision (cca 16 digits) by an elementary stepping algorithm is up to two orders of magnitude higher than is possible to obtain by Braak's solution. Any first $n$ eigenvalues of the Rabi model arranged in increasing order can be determined as zeros of $\phi_{N}(\epsilon)$ of at least the degree $N=n+n_{t}$. The value of $n_{t}>0$, which is slowly increasing with $n$, depends on the required precision. For instance, $n_{t} \simeq 26$ for $n=1000$ and dimensionless interaction constant $\kappa=0.2$, if double precision is required. Given that the sequence of the $l$ th zeros $x_{n l}$ 's of $\phi_{n}(\epsilon)$ 's defines a monotonically decreasing discrete flow with increasing $n$, the Rabi model is indistinguishable from an algebraically solvable model in any finite precision. Although we can rigorously prove our results only for dimensionless interaction constant $\kappa<1$, numerics and exactly solvable example suggest that the main conclusions remain to be valid also for $\kappa \geq 1$.
\end{abstract}

\section{INTRODUCTION}

Let us consider a quantum model described by a Hamiltonian $\hat{H}$ satisfying the eigenvalue equation

$$
\hat{H} \varphi=E \varphi
$$

in the Bargmann Hilbert space $\mathfrak{b}$ of analytic entire functions [1, 2]. The latter implies that any physical state is described by an entire function

$$
\varphi(z)=\sum_{n=0}^{\infty} \phi_{n} z^{n},
$$

where $\left\{\phi_{n}\right\}_{n=0}^{\infty}$ are the sought expansion coefficients. The present work investigates consequences of the following three simple observations.

First observation.- A first trivial observation is that for $\varphi$ to be an element of $\mathfrak{b}$, the coefficients $\phi_{n}$ 's have to approach zero in the limit $n \rightarrow \infty$. Hence for energy $\epsilon$ to belong to the spectrum $\Sigma, \phi_{n}$ have to be such a solution of Eq. (1) that $\phi_{n} \rightarrow 0$. Briefly,

$$
\epsilon \in \Sigma \Longrightarrow \phi_{n} \rightarrow 0 \quad(n \rightarrow \infty) .
$$

Obviously, the arrow cannot be reversed without some further limitations. There could be solutions of Eq. (11) going to zero in the limit $n \rightarrow \infty$ which need not lead to an entire function, and hence to an element of $\mathfrak{b}$ (e.g. $\left|\phi_{n}\right| \sim n^{-c}$, where $c$ is an arbitrary positive constant).

Second observation.- For a number of models [1, 3, [4], the eigenvalue equation (11) reduces in the Bargmann space $\mathfrak{b}$ to a three-term difference equation

$$
\phi_{n+1}+a_{n} \phi_{n}+b_{n} \phi_{n-1}=0 \quad(n \geq 0) .
$$

The recurrence coefficients $a_{n}$ and $b_{n}$ are functions of model parameters, and so are the coefficients $\phi_{n}$ 's. Our second observation regards the case when (i) $b_{n} \neq 0$ and (ii) the recurrence coefficients have at most an asymptotic power-like dependence

$$
a_{n} \sim a n^{\varsigma}, \quad b_{n} \sim b n^{v} \quad(n \rightarrow \infty),
$$

where $2 \varsigma>v$ and $\tau=\varsigma-v \geq 1 / 2$. The above conditions select an important class $\mathcal{R}$ of quantum models that was initially introduced and studied in our earlier work [3]. Prominent examples comprise a displaced harmonic oscillator [1, 3, 5], the Rabi model [6], two-mode squeezed harmonic oscillator [4], etc. The second observation is that, for the models of $\mathcal{R}$, also the reverse condition to that in Eq. (31) applies. Energy $\epsilon$ belongs to the spectrum $\Sigma$ if and only if $\phi_{n}(\epsilon) \rightarrow 0$ in the limit $n \rightarrow \infty$. We have the spectral condition

$$
\epsilon \in \Sigma \Longleftrightarrow \phi_{n}(\epsilon) \rightarrow 0 \quad(n \rightarrow \infty) .
$$

Indeed, according to the Perron-Kreuser theorem (Theorem 2.3 in Ref. [7]), there are possible two qualitatively different types of linearly independent solutions of the recurrence (4). The asymptotic behaviour of the minimal solution guaranteed by the Perron-Kreuser theorem is

$$
\frac{\phi_{n+1}}{\phi_{n}} \sim-\frac{b}{a} \frac{1}{n^{\tau}} \rightarrow 0 \quad(n \rightarrow \infty)
$$


[in virtue of Eq. (5) and $\tau \geq 1 / 2>0$ ]. On the other hand, the dominant solutions of the recurrence (4) behave as $\phi_{n+1} / \phi_{n} \sim-a n^{\varsigma}$ in the limit $n \rightarrow \infty$. For either (i) $\varsigma>0$ or (ii) $\varsigma=0$ and $a>1$ the absolute value of $\phi_{n}$ tends to infinity. The above dichotomy precludes any intermediate behaviour like $\left|\phi_{n}\right| \sim n^{-c}$. Consequently, any solution of the recurrence (4) with given initial conditions that behaves as $\phi_{n}(\epsilon) \rightarrow 0$ in the limit $n \rightarrow \infty$ corresponds necessarily to an eigenvalue $\epsilon \in \Sigma$.

Third observation.- Our third observation concerns the case when each of the expansion coefficients $\phi_{n}$ 's is proportional to a polynomial of degree $n$ in the energy parameter $\epsilon$ [5]. We recall that the necessary and sufficient condition for a family of polynomials $\left\{P_{n}\right\}$ (with degree $\left.P_{n}=n\right)$ to form an orthogonal polynomial system (OPS) is that $P_{n}$ 's satisfy

$$
P_{n}(x)=\left(\beta_{n} x-c_{n}\right) P_{n-1}(x)-\lambda_{n} P_{n-2}(x)
$$

with the initial condition $P_{-1}(x)=0$ and $P_{0}(x)=1$, where the coefficients $\beta_{n}, c_{n}$ and $\lambda_{n}$ are independent of $x, \beta_{n} \neq 0$, and $\lambda_{n} \neq 0$ for $n \geq 1$ [8]. In what follows, the Stieltjes measure $d \psi(x)$ in the orthogonality relations induced by the positive moment functional

$$
\begin{aligned}
\mathcal{L}\left[P_{m}(x) P_{n}(x)\right] & =\int_{-\infty}^{\infty} P_{m}(x) P_{n}(x) d \psi(x) \\
& =\lambda_{1} \lambda_{2} \ldots \lambda_{n+1} \delta_{m n}
\end{aligned}
$$

shall have a discrete support [5]. In other words the set of all points $x$ at which the resulting Stieltjes measure $d \psi(x) \equiv \psi(x)-\psi(x-0)>0$ forms a discrete lattice $\Lambda$ [8]. (In mathematics, the set $\Lambda$ is called the spectrum of $\psi$ - cf. p. 51 of Ref. [8].)

Orthogonal polynomials that are pairwise orthogonal with respect to a discrete measure are called discrete orthogonal polynomials [9, 10]. The requirement that the expansion coefficients $\phi_{n}$ 's are proportional to discrete orthogonal polynomials obviously entails a certain restriction on the form of the recurrence coefficients $a_{n}$ and $b_{n}$ of our initial recurrence (4). Nevertheless, the latter is still satisfied for a number of important models [5]. Canonical properties of an OPS are that $P_{n}$ 's

- have real and simple zeros (Theorem I-5.2 of Ref. [8]),

- the zeros of any two subsequent polynomials $P_{n}(x)$ and $P_{n+1}(x)$ mutually separate each other (Theorem I-5.3 of Ref. [8]).

Specifically, denote the zeros of $P_{n}(x)$ with degree $P_{n}=n$ by $x_{n 1}<x_{n 2}<\ldots<x_{n n}$. Then for any $l=1,2, \ldots$, $n-1$

$$
x_{n l}<x_{n-1, l}<x_{n, l+1} .
$$

For each fixed $l,\left\{x_{n l}\right\}_{n=l}^{\infty}$ is a strictly decreasing se- quence, defining a discrete flow, and the limit

$$
\lim _{n \rightarrow \infty} x_{n l}=\xi_{l} \in \Sigma
$$

exists [8]. Because of the spectral condition (6]), the discrete flow has nowhere to flow than to the spectral point of our model. Hence $\xi_{l} \in \Sigma$. In other words, the spectrum of a physical model coincides with the corresponding discrete lattice $\Lambda$.

Central questions.- The first two observations might have prompted the knowledgeable reader to pose the following question Q1: Could the zeros of a given $\phi_{n}(\epsilon)$ be of use to determine the spectrum of models from the recurrence class $\mathcal{R}$ ?

The very fact that the spectrum of a model is determined as zeros of a polynomial implies a special case of analytic solvability known as algebraic solvability 11 15]. Our another question is therefore Q2: Are there some models of the class $\mathcal{R}$ which are algebraically solvable?

The rest of the paper is devoted to answering the questions in the special case of the Rabi model [6].

\section{RABI MODEL}

The Rabi model [6] describes the simplest interaction between a cavity mode with a frequency $\omega_{c}$ and a twolevel system with a resonance frequency $\omega_{0}$. The model is characterized by the Hamiltonian [1, 6]

$$
\hat{H}_{R}=\hbar \omega_{c} \mathbb{1} \hat{a}^{\dagger} \hat{a}+\hbar g \sigma_{1}\left(\hat{a}^{\dagger}+\hat{a}\right)+\mu \sigma_{3},
$$

where $\mu=\hbar \omega_{0} / 2, \hat{a}$ and $\hat{a}^{\dagger}$ are the conventional boson annihilation and creation operators satisfying commutation relation $\left[\hat{a}, \hat{a}^{\dagger}\right]=1$, and $g$ is a coupling constant. In what follows, $\mathbb{1}$ is the unit matrix, $\sigma_{j}$ are the Pauli matrices in their standard representation, and we set the reduced Planck constant $\hbar=1$. The Hilbert space is $\mathcal{B}=L^{2}(\mathbb{R}) \otimes \mathbb{C}^{2}$, where $L^{2}(\mathbb{R})$ is represented by the Bargmann space of entire functions $\mathfrak{b}$, and $\mathbb{C}^{2}$ stands for a spin space [1, 2]. In a unitary equivalent single-mode spin-boson picture, $\hat{H}_{R}$ becomes

$$
\hat{H}_{s b}=\omega_{c} \mathbb{1} \hat{a}^{\dagger} \hat{a}+\mu \sigma_{1}+g \sigma_{3}\left(\hat{a}^{\dagger}+\hat{a}\right) .
$$

The transformation is accomplished by means of the unitary operator

$$
U=\frac{1}{\sqrt{2}}\left(\sigma_{1}+\sigma_{3}\right)=\frac{1}{\sqrt{2}}\left(\begin{array}{cc}
1 & 1 \\
1 & -1
\end{array}\right)=U^{-1}
$$

The Hilbert space can be written as a direct sum $\mathcal{B}=$ $\mathcal{B}_{+} \oplus \mathcal{B}_{-}$of the parity eigenspaces of the parity operator $\hat{\Pi}=\sigma_{1} \hat{\gamma}[3,5,16,17]$. Here $\hat{\gamma}=e^{i \pi \hat{a}^{\dagger} \hat{a}}$ induces reflections of the annihilation and creation operators: $\hat{a} \rightarrow-\hat{a}$, $\hat{a}^{\dagger} \rightarrow-\hat{a}^{\dagger}$, and leaves the boson number operator $\hat{a}^{\dagger} \hat{a}$ invariant [16, 17]. The corresponding parity eigenstates $\Phi^{+}$and $\Phi^{-}$of the eigenvalue equation (11) contain one 
independent component each [3, 5, 16, 17],

$$
\Phi^{+}(z)=\left(\begin{array}{c}
\varphi^{+} \\
\hat{\gamma} \varphi^{+}
\end{array}\right), \quad \Phi^{-}(z)=\left(\begin{array}{c}
\varphi^{-} \\
-\hat{\gamma} \varphi^{-}
\end{array}\right) .
$$

The respective parity eigenstates $\Phi^{+}(z)$ and $\Phi^{-}(z)$ satisfy the following eigenvalue equations for the independent (e.g. upper) component (cf. Eqs. (4.12-13) of Ref. [16])

$$
\begin{aligned}
& H^{+} \varphi^{+}=[A+B \hat{\gamma}+C] \varphi^{+}=E^{+} \varphi^{+}, \\
& H^{-} \varphi^{-}=[A-B \hat{\gamma}+C] \varphi^{-}=E^{-} \varphi^{-},
\end{aligned}
$$

where $A=\omega_{c} \hat{a}^{\dagger} \hat{a}, B=\mu$, and $C=g\left(\hat{a}^{\dagger}+\hat{a}\right)$. Here we have written $E^{ \pm}$since, in general, the spectra of $H^{+}$and $H^{-}$do not coincide.

Now, in the Bargmann space of entire functions, the action of $\hat{\gamma}$ becomes (Eq. (10) of Ref. [5]; Eq. (37) of Ref. [3])

$$
\hat{\gamma} \varphi^{ \pm}(z)=\varphi^{ \pm}(-z)=\sum_{n=0}^{\infty}(-1)^{n} \phi_{n}^{ \pm} z^{n} .
$$

Thereby, the Rabi model can be characterized by a pair of the three-term recurrences (Eq. (37) of Ref. [3])

$$
\begin{array}{r}
\phi_{n+1}^{ \pm}+\frac{1}{\kappa(n+1)}\left[n-\epsilon \pm(-1)^{n} \Delta\right] \phi_{n}^{ \pm} \\
+\frac{1}{n+1} \phi_{n-1}^{ \pm}=0,
\end{array}
$$

where $\epsilon \equiv E^{ \pm} / \omega_{c}, \Delta=\mu / \omega_{c}=\omega_{0} /\left(2 \omega_{c}\right)$, and $\kappa=g / \omega_{c}$ reflects the coupling strength [3]. Because the recurrence (16) satisfies the conditions that guarantee uniqueness of the minimal solution, i.e. each $\varphi^{ \pm}(z)$ generated by the respective minimal solutions is unique, the spectrum in each parity eigenspace $\mathcal{B}_{ \pm}$is necessarily nondegenerate (cf. sec. 5.2 of Ref. [5]).

As shown in our recent work [5], the substitution $\phi_{n}^{ \pm}(\epsilon)=P_{n}^{(-1)}(x) / n$ ! transforms each of the two threeterm recurrences (16) into the defining equation of monic orthogonal polynomials [cf. Eq. (8)],

$$
\begin{aligned}
& P_{n}^{(\alpha)}(x)=\left(x-c_{n+\alpha}\right) P_{n-1}^{(\alpha)}(x)-\lambda_{n+\alpha} P_{n-2}^{(\alpha)}(x), \\
& P_{-1}^{(\alpha)}(x)=0, \quad P_{0}^{(\alpha)}(x)=1,
\end{aligned}
$$

where $\alpha=-1, x=\epsilon / \kappa$,

$$
c_{n} \equiv \frac{1}{\kappa}\left[n \pm(-1)^{n} \Delta\right],
$$

$\lambda_{n}=n$ for $n>0$, and $\lambda_{0}=1$ [5]. Note that the coefficients $c_{n}$ and $\lambda_{n}$ are real and independent of $x$, and $\lambda_{n+\alpha}>0$ for $n \geq 1$. Because the Stieltjes measure $d \psi(x)$ in the orthogonality relations (9) has a discrete support [5], Eq. (9) reduces to

$$
\begin{aligned}
\mathcal{L}\left[P_{m}(x) P_{n}(x)\right]= & \sum_{x_{i} \in \Lambda} P_{m}^{(-1)}\left(x_{i}\right) P_{n}^{(-1)}\left(x_{i}\right) d \psi\left(x_{i}\right) \\
& =n ! \delta_{m n},
\end{aligned}
$$

where $\Lambda$ is a one-dimensional lattice representing the discrete support of $d \psi(x)$. Therefore, the resulting polynomials are discrete orthogonal polynomials [9, 10, 18, 19]. One can verify that, except for the special limiting case $\Delta=0$ (discussed below), the polynomials are nonclassical orthogonal polynomials (i.e. they cannot be recovered as solution of a second-order difference equation of hypergeometric type - cf. Secs. 2-3 of Ref. [9]; the classical polynomials are called the Hahn class of orthogonal polynomials in Sec. V-3 of Ref. [8]).

The three-term recurrences (16) imply that the exponents $\varsigma=0, v=-1$ and $\tau=\varsigma-v=1 \geq 1 / 2$ [cf. Eq. (5)]. Therefore, the conditions required for the validity of our first to third observations are satisfied for the Rabi model, provided that $\kappa<1$. The above range encompasses not only the conventional strong coupling regime characterized in that $\kappa=g / \omega_{c} \lesssim 10^{-2}$ but also the ultrastrong $(\kappa \gtrsim 0.1)$ coupling regime, and overlaps with the deep strong $(\kappa \approx 1)$ coupling regime [20]. For $\kappa \gtrsim 0.1$ the validity of the rotating wave approximation (RWA) breaks down and the relevant physics can only be described by the full Rabi model [6] .

\section{MAIN RESULTS}

Let us elucidate our main results on the example of the exactly solvable limit $\Delta=0$ describing a displaced harmonic oscillator [1]. The expansion coefficients $\phi_{n}$ are known to be determined by the associated Laguerre polynomials $L_{n}^{(\zeta-n)}\left(\kappa^{2}\right)$, where $\zeta=\epsilon+\kappa^{2}=\kappa x+\kappa^{2}$. (cf. Eq. (2.16) of Ref. [1] and Sec. 4 of Ref. [5]). Note in passing that energy variable $\zeta$ is not the polynomial variable of the associated Laguerre polynomials. It is expedient to work with the (monic) Charlier polynomials [21] (cf. Eqs. VI-1.4-5 of Ref. [8]) and express $\phi_{n}$ as (see Sec. 4 of Ref. [5])

$$
\phi_{n}(\epsilon)=\frac{P_{n}^{(-1)}(x ; \Delta=0)}{n !}=\frac{1}{n ! \kappa^{n}} C_{n}^{\left(\kappa^{2}\right)}(\zeta) .
$$

The Stieltjes measure $d \psi^{\left(\kappa^{2}\right)}$ in the orthogonality relations of the Charlier polynomials (cf. Eq. VI-1.3 of Ref. [8]) is known to be the step function

$$
d \psi^{\left(\kappa^{2}\right)}(\zeta)=\sum_{l=0}^{\infty} \frac{e^{-\kappa^{2}} \kappa^{2 \zeta}}{\zeta !} \delta(\zeta-l) .
$$

Note in passing that $d \psi^{\left(\kappa^{2}\right)}$ is the Poisson distribution function of probability theory at the jumps [8]. The jumps occur at $\zeta=0,1,2, \ldots$ The set of all the jumps forms the support of the Stieltjes measure $d \psi^{\left(\kappa^{2}\right)}$ [8], which in turn is known to be formed by the set of all the limit zero points $\xi_{l}$ defined earlier by Eq. (11) [8]. 

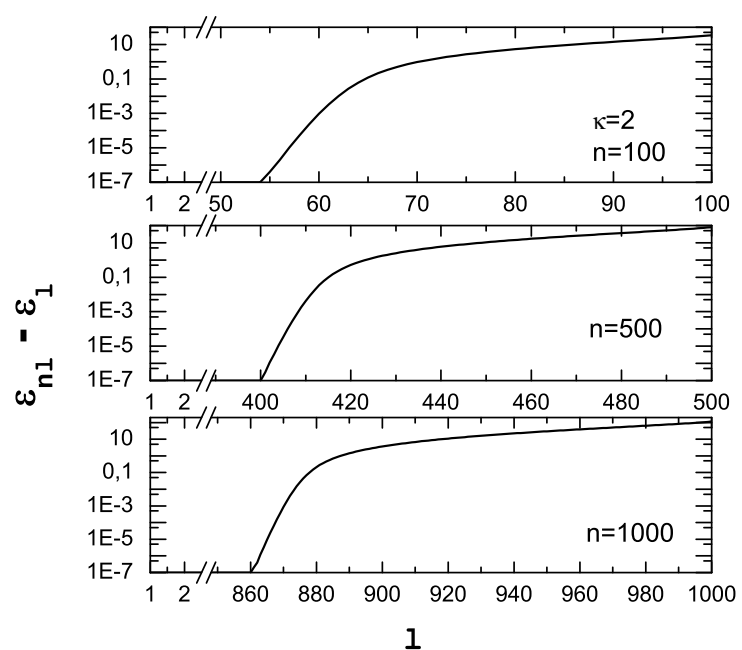

1

Figure 1. An illustration of the approximation of the spectrum in the case of the exactly solvable displaced harmonic oscillator, which corresponds to the Rabi model in the limit $\Delta=0$. Shown is the difference of the approximants determined by the zeros $x_{n l}, l=1,2, \ldots, n$, of $\phi_{n}(\epsilon)$ compared to the exact eigenvalues $\epsilon_{l-1}=l-1-\kappa^{2}$ for $\kappa=2$ and different degree $n$ of $\phi_{n}(\epsilon)$. The precision in calculating zeros was set to seven decimal places.

Thus the orthogonality relations are

$$
\sum_{l=0}^{\infty} C_{m}^{\left(\kappa^{2}\right)}(l) C_{n}^{\left(\kappa^{2}\right)}(l) d \psi^{\left(\kappa^{2}\right)}(l)=\kappa^{2 n} n ! \delta_{m n}
$$

Not surprizingly, the location of jumps correspond exactly to the eigenvalues of the displaced harmonic oscillator [1]

$$
\epsilon_{l}=l-\kappa^{2}
$$

(including $l=0$ ). The jumps define an equidistant lattice. Because the orthogonality relation (22) reduces to an infinite sum, the Charlier polynomials are said to be classical discrete orthogonal polynomials on an equidistant lattice [9, 10, 18, 19]. The adjective classical implies that the Charlier polynomials can be recovered as solutions of a second-order difference equation of hypergeometric type (cf. Sec. 2 of Ref. [9]).

Thus in the example of the displaced harmonic oscillator our conclusions can be shown to be rigorously valid also for $\kappa \geq 1$. As a by-product, none of the zeros of $\phi_{n}$ coincides with the exact spectrum. In more detail, Eq. (74) of Ref. [5] shows that $\phi_{n}$ is a sum of polynomials in the dimensionless energy parameter $\zeta$,

$$
\phi_{n}=\sum_{j=0}^{n}(-1)^{n-j} \frac{\kappa^{n-2 j}}{(n-j) ! j !} \prod_{k=0}^{j-1}(\zeta-k) .
$$

The spectral points $\zeta=l \in \mathbb{N}$ (including $l=0$ ) of the displaced harmonic oscillator are characterized by a sudden collapse of the degree of $\phi_{n}$ to a polynomial of merely the degree $(l-1)$ in $\zeta$ for any $n \geq l[5]$. Consequently, $\phi_{n}$ reduces for any $\zeta=l$ to a finite sum of $l$ terms, each ranging from $(-1)^{n} \kappa^{n} / n$ ! for $j=0$ to the $j=(l-1)$ th term

$$
(-1)^{n+1-l} \frac{l \kappa^{n+2-2 l}}{(n+1-l) !} .
$$

Clearly, the points of the spectrum $\zeta=l$ do not coincide with the zeros of any of $\phi_{n}$. However, each of the individual terms rapidly decreases with increasing $n$ in its absolute value down to zero. It is straightforward to show that for any $\zeta=l \in \mathbb{N}$ the absolute value of $\phi_{n}$ could be bounded by $l^{2} \max \left(\kappa^{n}, \kappa^{n+2-2 l}\right) /(n+1-l) !$. Hence

$$
\phi_{n}(\zeta=l) \rightarrow 0 \quad(n \rightarrow \infty),
$$

i.e., at any given point of the spectrum $\phi_{n}$ rapidly vanishes in the limit $n \rightarrow \infty$ down to zero (cf. figure 1).

For a nonzero value of $\Delta$ our polynomials cannot be recovered as solution of a second-order difference equation of hypergeometric type (cf. Secs. 2-3 of Ref. 9]). Thus $\Delta \neq 0$ induces a deformation of the Charlier polynomials to non-classical discrete orthogonal polynomials and, at the same time, a deformation of the underlying equidistant lattice. Although neither the weight function nor the deformed lattice are analytically known, the orthogonality relations (9) enable us to conclude that the above deformation is a norm preserving deformation. Indeed, Eq. (9) implies that the norm depends only on the value of the recurrence coefficients $\lambda_{j}$. However, the latter do not depend on $\Delta$. Thus, as exemplified by Eq. (19), $\left\|P_{n}^{(-1)}\right\|^{2}=n$ ! for any value of $\Delta$ [cf. Eqs. (20) and (22)].

Regarding the question $\mathbf{Q} 1$ raised in the preceding section, the answer turns out thus not only be affirmative, but the result exceeds all the expectations also in the case of the Rabi model (cf. figure 2). The convergence of the zeros to the spectrum is very fast. The tail of highestorder zeros which do not approximate the spectrum increases for a given $n$th level with increasing $\kappa$. Although we can rigorously prove our results only for $\kappa<1$, numerics strongly suggests that the main conclusions remain to be valid also for $\kappa \geq 1$. Convergence of each discrete flow of zeros has been independently checked by the Schweber quantization criterion [1, 3, [5] (see also Sec. IVB below). The example in figure 2 shows that the fraction $n_{t} / \kappa=(n-1000) / \kappa$ saturates at some constant value for $\kappa \gtrsim 1$. The eigenstate corresponding to the $n$th eigen- 


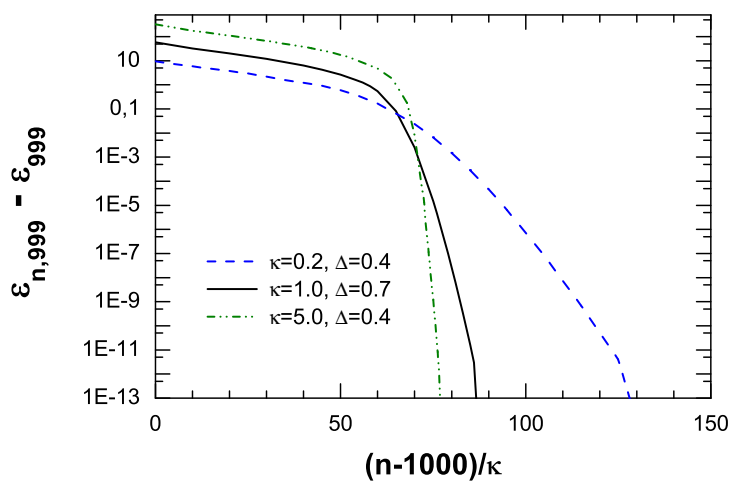

Figure 2. Convergence of the 1000th zero $\epsilon_{n, 999}$ of $\phi_{n}(\epsilon)$ toward the exact 1000th eigenvalue $\epsilon_{999}=$ 998.907883759510, 997.950425260357 and 973.989087026621 of the Rabi model in the positive parity eigenspace for $(\kappa, \Delta)=(0.2,0.4),(1,0.7),(5,0.4)$, respectively. The ground state energy $\epsilon_{0}$ corresponds to the first zero of $\phi_{n}(\epsilon)$. Each decreasing sequence $\epsilon_{n, 999}$ forms a discrete flow converging toward the corresponding exact eigenvalue down to machine precision.

value can be approximated for $N=n+n_{t}$ as

$$
\varphi(z) \approx \sum_{l=0}^{N} \phi_{l}\left(x_{N n}\right) z^{l}+\sum_{l=N+1}^{\infty} \frac{(-\kappa z)^{l}}{l !}
$$

In agreement with the asymptotic behaviour of the $m i$ nimal solution enforced by the Perron-Kreuser theorem (7), $\phi_{n}(\epsilon)$ has to behave for any eigenvalue as $\phi_{n}(\epsilon) \sim$ $(-\kappa)^{n} / n$ ! for sufficiently large $n$ (note that the three-term recurrences (16) implies $a=1 / \kappa, \varsigma=0, b=1, v=-1$ ).

By the well known relations connecting the zeros and coefficients of a polynomial (Theorems I-4.2 and IV-3.1 of Ref. [8])

$$
\sum_{l=0}^{n-1} \epsilon_{l} \approx \kappa \sum_{l=1}^{n} c_{l}=\frac{n(n-1)}{2}+d
$$

where we have substituted from (18) for $c_{l}$, and $d$ is one of $0, \pm \Delta$. The latter justifies that energy eigenvalues are rather closely distributed around a straight line [22].

The answer to our question Q2 appears peculiar. Our computers allows us to work only in a finite precision. However, in the given precision, the spectrum of the Rabi model can be determined by the zeros of the polynomials $\phi_{n}(\epsilon)$. We have seen above that the discrete zeros flow has nowhere to flow than to the spectral point (cf. figure 2). Therefore, in any finite precision the Rabi model is indistinguishable from an algebraically solvable model. At the same time, only a computer with unlimited precision would recognize that the Rabi model is not algebraically solvable, because the limit $n \rightarrow \infty$ is required for the zeros flow to converge to the spectrum. Note in passing (see below) that the same limit is also required in Braak's solution.

\section{DISCUSSION}

Solving the spectral condition (6) implies an entirely new, efficient, and relatively general method in determining the spectrum. The method differs both from (i) a brute force numerical diagonalization, (ii) searching for zeros of functions determined by infinite continued fractions as in the Schweber method (cf. Eq. (A.16) of Ref. [1]), and (iii) Braak's approach. Only the lowest 10-20 energy levels are within the reach of both Braak's solution [23] and, as shown below, of the Schweber method [1, 3] - you are invited to convince yourself by running numerical F77 code that has been made available on-line [24]. A brute force numerical diagonalization allows one to determine above 2000 energy levels in double precision (cca 16 digits). However any deeper analytic insight is missig. Note in passing that the presently calculable 1350 energy levels per parity subspace have been obtained by the simplest stepping algorithm. Then the numerical limitation in calculating zeros are over- and underflows. Typically, with increasing $n$ the respective recurrences yield first increasing and then decreasing $\phi_{n}$. It is conceivable that the use of a more sophisticated algorithm could overcome the limit of the total number of calculable energy levels of ca. 1350 levels per parity subspace, or ca 2700 levels for the Rabi model in total, in double precision.

That expansion coefficients $\phi_{n}$ could be determined by orthogonal polynomials is, strictly speaking, not necessary for working of our method based on solving the spectral condition (6). The method could provide also an efficient numerical way of obtaining the spectra of the models which expansion coefficients $\phi_{n}$ cannot be given by orthogonal polynomials.

\section{A. Comparison with Braak's solution}

Braak 17] argued that a regular spectrum of the Rabi model in the respective parity eigenspaces is given by the zeros of transcendental functions

$$
G_{ \pm}(\zeta)=\sum_{n=0}^{\infty} K_{n}(\zeta, \kappa)\left[1 \mp \frac{\Delta}{\zeta-n}\right] \kappa^{n} .
$$

The coefficients $K_{n}(\zeta, \kappa)$ are obtained recursively by solving the Poincaré difference equation

$$
K_{n+1}-\frac{f_{n}(\zeta)}{(n+1)} K_{n}+\frac{1}{n+1} K_{n-1}=0
$$


upwardly for $n \geq 1$, where

$$
f_{n}(\zeta)=2 \kappa+\frac{1}{2 \kappa}\left(n-\zeta-\frac{\Delta^{2}}{n-\zeta}\right),
$$

$\kappa$ and $\Delta$ are as in Eq. (16) (cf. Eq. (A8) of Schweber [1], which has mistyped sign in front of his $b_{n-1}$, and Eqs. (4) and (5) of [17]). The initial condition is

$$
K_{1} / K_{0}=f_{0}(\zeta)=2 \kappa-\frac{1}{2 \kappa}\left(\zeta-\frac{\Delta^{2}}{\zeta}\right),
$$

with $K_{0}$ being a normalization constant. Braak's solution requires (i) to solve for an undetermined number of complicated functions $K_{n}(\zeta, \kappa)$ having poles at discrete values of $\zeta$ (cf. Sec. 5.1 of Ref. [5]), (ii) to assemble the functions $K_{n}(\zeta, \kappa)$ into $G_{ \pm}(\zeta)$ according to Eq. (27), (iii) to solve for zeros of $G_{ \pm}(\zeta)$. Thus it is not surprizing that Braak's approach reaches its limits already at ca. 20 energy levels in double precision [23]. Even if an additional analytic continuation step could increase the number of calculable energy levels in Braak's approach to around 100 [23], the number is still by an order of magnitude lower than what is possible within our approach.

In contrast, in our approach the structure of any $\phi_{n}(\epsilon)$ 's is clear - they are all determined by orthogonal polynomials. Further, only a single well-behaved $\phi_{N}(\epsilon)$ of the degree $N=n+n_{t}, n_{t}>0$, is required to determine any first $n$ eigenvalues of the Rabi model arranged in increasing order as zeros of $\phi_{N}(\epsilon)$. Importantly, our approach also provides an efficient registry of energy levels. Indeed, a given $\phi_{n}(\epsilon)$ has $n$ distinct real zeros. Therefore any omission of energy level can be easily identified. The latter could be useful in any future statistical analysis of the spectra [22]. To reach unlimited precision, the limit $n \rightarrow \infty$ is required. However, the latter is also necessary in the definition of $G_{ \pm}(\zeta)$.

\section{B. Failure of Schweber's method for higher order eigenvalues}

It is believed that the spectrum of the Rabi model can be formally determined by the Schweber quantization criterion expressed in terms of infinite continued fractions (cf. Eq. (A.16) of Ref. [1] and Refs. [3, 5]),

$$
0=F(x) \equiv a_{0}+\frac{-b_{1}}{a_{1}-} \frac{b_{2}}{a_{2}-} \frac{b_{3}}{a_{3}-} \cdots,
$$

where [cf. Eq. (16)]

$$
a_{n}=\frac{1}{\kappa(n+1)}\left[n-\epsilon \pm(-1)^{n} \Delta\right], \quad b_{n}=\frac{1}{n+1},
$$

According to the Wallis formulas (Eqs. (III.2.1) of Ref. [8]; Eqs. (4.2-3) of Ref. 7]), the infinite continued frac- tion in Eq. (30) can be expressed as the limit

$$
r_{0}=\lim _{n \rightarrow \infty} \frac{A_{n}}{B_{n}} .
$$

Here $A_{n}$ and $B_{n}$ are the $n$th partial numerator and the $n$th partial denominator, respectively. We have shown that the ratio on the r.h.s. of Eq. (32), also known as a convergent, can be expressed as the limit of the ratios of the polynomials [5]

$$
r_{0}=\lim _{n \rightarrow \infty} \frac{P_{n-1}^{(1)}(x)}{P_{n}(x)}
$$

where $P_{n}^{(\alpha)}$ satisfy Eq. (17) for $\alpha=0,1$. The $n$th partial numerator $A_{n}$ in Eq. (32) is related to $P_{n-1}^{(1)}(x)$, whereas the $n$th partial denominator $B_{n}$ is related to $P_{n}(x)$. Any numerical method of computing $F(x)$ through Eqs. (32) and (33) has to impose an unavoidable cutoff at some $n=N \gg 1$. For any finite $n$ the ratio in (33) enables the partial fraction decomposition (PFD) (Theorem III-4.3 of Ref. [8]),

$$
\frac{A_{n}}{B_{n}}=\frac{P_{n-1}^{(1)}(x)}{P_{n}(x)}=\sum_{l=1}^{n} \frac{M_{n l}}{x-x_{n l}},
$$

where the numbers $M_{n l}$ are all positive and satisfy the condition $\sum_{l=1}^{n} M_{n l}=1[\underline{5}, 8]$.

Let $F_{n}(x)$ denote a finite-order approximation to $F(x)$ defined by Eq. (30), which is obtained by approximating $r_{0}$ in Eqs. (32) and (33) by the PFD in Eq. (34). One finds $d F_{n}(x) / d x<0$ whenever the derivative exists [5]. Consequently, $F_{n}(x)$ decreases from $+\infty$ to $-\infty$ between any two subsequent $x_{n l}<x_{n, l+1}$ and there is exactly one zero of $F_{n}(x)$ [5] (cf. figs. 1,2 of Ref. [3] and fig. 1 of Ref. [5]). $F_{n}(x)$ has its zeros and poles interlaced on the real axis [5]. As is the case of any associated OPS's (see sec. III.4 of Ref. [8]), the zeros of $P_{n}^{(\alpha)}(x)$ and $P_{n-1}^{(\alpha+1)}(x)$ are interlaced (Theorem III-4.1 of Ref. [8]). Specifically,

$$
x_{n l}^{(\alpha)}<x_{n-1, l}^{(\alpha+1)}<x_{n, l+1}^{(\alpha)}, \quad \alpha=-1,0 .
$$

(It is reminded that the superscript $\alpha=-1$ denotes the zeros of $\phi_{n}$ 's.) The second of the rigorous sharp inequalities in Eq. (35) implies

$$
x_{n-1, l-1}^{(1)}<x_{n l}<x_{n+1, l+1}^{(-1)} .
$$

(For the sake of notation the superscript (0) for $\alpha=0$ will be suppressed in what follows.) The above scenario can be indeed confirmed numerically for a small number of the very first eigenvalues (cf. figs. 1,2 of Ref. [3]; fig. 1 of Refs. [5, 25]).

Nevertheless, any practical implementation of the Schweber method fails for higher order eigenvalues. Depending on the model parameters, one can determine only up to 10-20 eigenvalues, and that already in the 


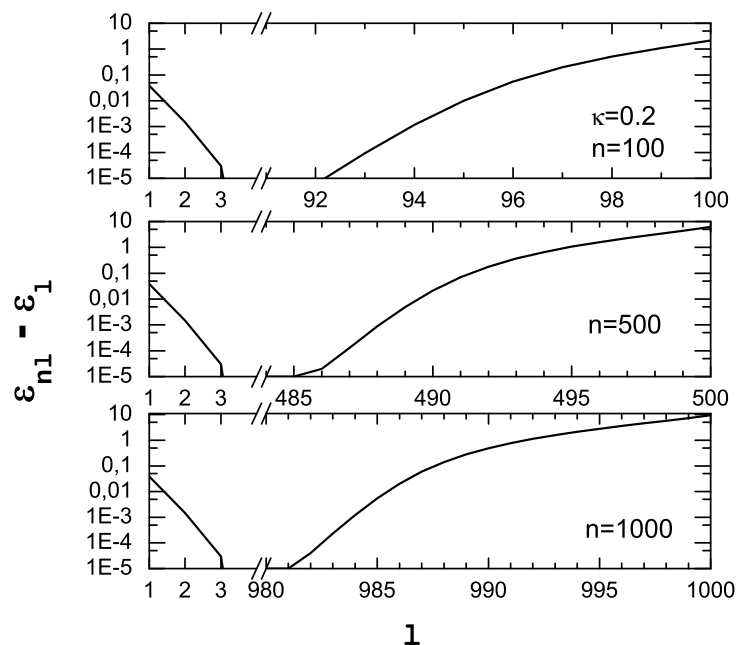

Figure 3. An approximation of the spectrum in the case of the exactly solvable displaced harmonic oscillator by the zeros $x_{n l}, l=1,2, \ldots, n$, of the partial denominators $B_{n}$ 's [cf. Eq. (32)]. Similar to figure 1 shown is the difference of the approximants $\epsilon_{n l}$ compared to the exact excited eigenvalues $\epsilon_{l}=l-\kappa^{2}, l>0$, for $\kappa=0.2$ and different degree $n$ of $B_{n}$. The ground state energy $\epsilon_{0}$ at $l=0$ is not accounted for by the OPS for $\alpha=0$. The precision in calculating zeros was set to five decimal places.

exactly solvable limit of the displaced harmonic oscillator - cf. F77 code made available online [24]. We have traced the failure down to a curious property of zeros of associated OPS - cf. data files [27]. Surprisingly enough, after a first few of initial zeros [e.g. beginning with $l \gtrsim 2$ for $(\kappa, \Delta)=(0.2,0.4)]$ one finds that, in spite of the strict inequalities (36),

$$
x_{n-1, l-1}^{(1)} \simeq x_{n l} \simeq x_{n+1, l+1}^{(-1)} .
$$

For $l \gtrsim 4$ and $(\kappa, \Delta)=(0.2,0.4)$ the zeros then coincide up to more than five decimal places (provided that $n$ is sufficiently large) - cf. data files [27]. Because of the coagulation of zeros (37), any singularity of $F_{n}(x)$ becomes numerically invisible. The coagulation can be undertood in that the position of zeros for each the OPS's is largely determined by the respective sequences

$$
q_{n}^{(\alpha)}(x)=\frac{\lambda_{n+\alpha}}{\left(x-c_{n+\alpha}\right)\left(x-c_{n+\alpha+1}\right)} .
$$

However, in the present case one finds that

$$
q_{n-\alpha}^{(\alpha)}(x)=\frac{\kappa^{2}(n+1)}{\left(n-\epsilon \pm(-1)^{n} \Delta\right)\left(n+1-\epsilon \mp(-1)^{n} \Delta\right)}
$$

do not depend on the value of $\alpha$. The coagulations of zeros is demonstrated in figure 3. The latter shows that it is possible to determine a large part of the spectrum by looking at the zeros of the partial denominators $B_{n}$ 's. Only marginally worse approximation property show the zeros of the partial numerators $A_{n}$ 's (not shown). We emphasize that the failure of Schweber's method is entirely down to the finite precision of numerical calculations. Although the rigorous theory underlying Schweber's method [1, 3, 5, 25] is perfectly valid, a practical value of the method may be thus rather limited. The above conclusions are expected to apply also to alternative continued fraction expressions for the Rabi model studied by Ziegler [26].

\section{Algebraic solvability}

The Rabi model is a typical example of quasi-exactly solvable (QES) models in quantum mechanics [11-15]. The QES models are distinguished by the fact that, for a chosen set of model parameters, a finite number of their eigenvalues and corresponding eigenfunctions can be determined algebraically [11 15]. In the case of the Rabi model [6], the latter eigenvalues correspond to the $J u d$ dian exact isolated solutions [28, 29]. The possibility of any other polynomial solution can be excluded by recent results of Zhang [30] (see also Sec. 4.2 of Ref. 5]). In agreement with the no-go theorem of Zhang [30], none of the zeros of any polynomial from any of the OPS $\left\{P_{n}^{(\alpha)}(x)\right\}$ coincides with the spectrum. The spectral points could only be reached in the limit $n \rightarrow \infty$.

Although the very notion of quantum integrability is the subject of ongoing dispute [31], it is largely accepted that if eigenvalues can be determined algebraically 1114], this implies integrability and solvability [31]. Our results indicate that the algebraic solvability could be intricately linked with available precision. Conceptually, and from a broader perspective, the above properties of the QES Rabi model provide an example of that, numerically, there may be only very subtle difference between exactly and quasi-exactly solvable models, if the latter are characterized by discrete orthogonal polynomials. In general, any (i.e. not necessary QES) model that satisfies the spectral condition (6) and is characterized by discrete orthogonal polynomials could exhibit such a solvability.

\section{Relation to the Jaynes and Cummings model and the effect of the RWA}

For dimensionless coupling strength $\kappa=g / \omega_{c} \lesssim 10^{-2}$, the physics of the Rabi model is known to be well captured by the analytically solvable Jaynes and Cummings (JC) model [32]. The latter is obtained from the former upon applying the rotating wave approximation (RWA), whereby the coupling term $\sigma_{1}\left(\hat{a}^{\dagger}+\hat{a}\right)$ in Eq. (12) is replaced by $\left(\sigma_{+} \hat{a}+\sigma_{-} \hat{a}^{\dagger}\right)$, where $\sigma_{ \pm} \equiv\left(\sigma_{1} \pm i \sigma_{2}\right) / 2$. The eigenstates of the JC model are linear combinations of 
the product states $\left|\psi_{1 n}\right\rangle=|n\rangle|e\rangle$ and $\left|\psi_{2 n}\right\rangle=|n+1\rangle|g\rangle$ in the Hilbert space $\mathcal{B}=L^{2}(\mathbb{R}) \otimes \mathbb{C}^{2}$, where the respective $|e\rangle=(1,0)^{t}$ and $|g\rangle=(0,1)^{t}$, with the superscript $t$ indicating the transpose, stand for the excited and ground state in the spin space $\mathbb{C}^{2}$ [1, 32]. The product states $\left|\psi_{1 n}\right\rangle$ and $\left|\psi_{2 n}\right\rangle$ form a basis of an invariant subspace of the operator

$$
\hat{J}=\mathbb{1} \hat{a}^{\dagger} \hat{a}+\frac{1}{2}\left(\mathbb{1}+\sigma_{3}\right),
$$

which generates a continuous $U(1)$ symmetry of the JC model [17, 32]. The invariant subspace is characterized by the eigenvalue $n+1$ of $\hat{J}$. In terms of the parity operator $\Pi=-\exp (i \pi \hat{J})$, each invariant subspace of $\hat{J}$ is positive or negative parity subspace depending on if $n$ is even or odd, respectively. Therefore, in each invariant subspace of $\hat{J}$, and hence for the eigenstates of the JC model, the parameter $w= \pm(-1)^{n} \Delta$ in Eq. (16) reduces to $w=\Delta$.

If $\kappa$ becomes small, the solution of the JC model [32] suggests to arrive at approximate solutions of Eq. (16) by setting all but two subsequent expansions coefficients $\phi_{l}$ and $\phi_{l+1}$ to zero. By forming corresponding $\varphi_{ \pm}(z)=$ $\phi_{l} z^{l}+\phi_{l+1} z^{l+1}$ according to Eq. (2), substituting into Eq. (15), and unitary transforming by the operator $U$ given by Eq. (14), one can verify that the corresponding parity eigenstates $\Phi^{+}$and $\Phi^{-}$[cf. Eq. (15)] in the single-mode boson picture $\hat{H}_{s b}$ become

$$
\Phi(z)=\left(\begin{array}{c}
\phi_{l} z^{l} \\
\phi_{l+1} z^{l+1}
\end{array}\right)
$$

in the conventional representation $\hat{H}_{R}$. Here we have used that $\Phi$ derives from the positive or negative parity eigenstate depending on if $l$ is even or odd, respectively. Note in passing that $\Phi(z)$ is yet undetermined linear combination of the JC states $\left|\psi_{1 l}\right\rangle$ and $\left|\psi_{2 l}\right\rangle$ in a given invariant subspace of $\hat{J}$. Obviously, upon imposing RWA onto $\hat{H}_{R}$ in Eq. (12) and substituting our $\Phi(z)$ as trial wave functions one would recover the JC model solution.

In order to investigate the effect of the RWA on the exact solution, we determine the eigenvalues of the JC model from the exact equations. Upon considering Eq. (16) for $n=l$ and $n=l+1$ one arrives at

$$
\begin{aligned}
\frac{\phi_{l+1}}{\phi_{l}} & =-\frac{1}{\kappa(l+1)}[l-\epsilon+\Delta], \\
\frac{\phi_{l}}{\phi_{l+1}} & =-\frac{1}{\kappa}[l+1-\epsilon-\Delta] .
\end{aligned}
$$

One can recast Eqs. (38) in the matrix form

$$
\left(\begin{array}{cc}
l+\Delta & (l+1) \kappa \\
\kappa & l+1-\Delta
\end{array}\right)\left(\begin{array}{c}
\phi_{l} \\
\phi_{l+1}
\end{array}\right)=\epsilon\left(\begin{array}{c}
\phi_{l} \\
\phi_{l+1}
\end{array}\right) .
$$

The secular equation reduces to a quadratic equation

$$
\epsilon^{2}-(2 l+1) \epsilon+l(l+1)+\Delta-\Delta^{2}-\kappa^{2}(l+1)=0 .
$$

The eigenvalues are

$$
\epsilon_{ \pm}=l+\frac{1}{2} \pm \frac{1}{2} \sqrt{1-4 \Delta+4 \Delta^{2}+4 \kappa^{2}(l+1)} .
$$

Given $\Delta=\omega_{0} /\left(2 \omega_{c}\right)$, one finds

$$
1-4 \Delta+4 \Delta^{2}=1-\frac{2 \omega_{0}}{\omega_{c}}+\frac{\omega_{0}^{2}}{\omega_{c}^{2}}=\frac{\left(\omega_{0}-\omega_{c}\right)^{2}}{\omega_{c}^{2}}=\delta_{c}^{2},
$$

i.e. square of the normalized detuning parameter $\delta_{c}=$ $\left(\omega_{0}-\omega_{c}\right) / \omega_{c}$ of the JC model [32]. Note in passing that $\delta_{c} \ll 1$ in the RWA, because the latter is reliable only if $g\left|\omega_{0}-\omega_{c}\right| \ll \omega_{0}, \omega_{c}$. The eigenvalues can be thus recast as

$$
\epsilon_{ \pm}=l+\frac{1}{2} \pm \frac{1}{2} \sqrt{\delta_{c}^{2}+4 \kappa^{2}(l+1)}
$$

which is the familiar form of the eigenvalues of the JC model [32].

Any exact regular solution of the Rabi model is characterized by infinite set of nonzero expansion coefficients $\phi_{n}$, which for sufficiently large $n$ behave as $\phi_{n} \sim$ $(-\kappa)^{n} / n$ ! [cf. the Perron-Kreuser theorem (7) and the recurrence Eq. (16)]. Interestingly, the RWA takes implicitly into account the effect of $\phi_{n} \neq 0$ for $n \neq l, l+1$. If the coefficients were ignored, Eq. (16) for $n=l-1$ and $n=l+2$ would require that additionally

$$
\phi_{l}=0, \quad \phi_{l+1} /(l+3)=0 .
$$

Afterwards one would find for $\epsilon=\epsilon_{+}$

$$
\frac{\phi_{l+1}}{\phi_{l}}=\tan \frac{\theta}{2}=\frac{2 \kappa}{D+\delta_{c}}=\frac{D-\delta_{c}}{2(l+1) \kappa},
$$

where we have substituted from (39) for $\epsilon$, denoted $D=\sqrt{\delta_{c}^{2}+4 \kappa^{2}(l+1)}$, and used that $\Delta-(1 / 2)=\delta_{c} / 2$. Because

$$
\tan \theta=\frac{2 \tan \frac{\theta}{2}}{1-\tan ^{2} \frac{\theta}{2}}
$$

and

$$
\tan ^{2} \frac{\theta}{2}=\frac{D-\delta_{c}}{D+\delta_{c}} \frac{1}{(l+1)},
$$

one can determine $\tan \theta$ as

$$
\tan \theta=\frac{4 \kappa}{D+\delta_{c}} \frac{(l+1)\left[D+\delta_{c}\right]}{l D+(l+2) \delta_{c}}=\frac{4(l+1) \kappa}{l D+(l+2) \delta_{c}} .
$$

The latter would not coincide with $\tan \theta=2 \kappa \sqrt{l+1} / \delta_{c}$ for the JC model solution [32]. Hence if one tries from the very outset to ignore in the exact solution all but a pair of expansion coefficients, one will arrive at the RWA energies but not to the RWA tangent value. 


\section{E. Open problems}

In Sec. II it has been alluded to that our polynomials underlying the Rabi model are non-classical discrete orthogonal polynomials [9, 10]. Various generalizations of the classical discrete orthogonal polynomials have been studied in the literature. However, they have been almost exclusively concerned with various generalizations of the Stieltjes weight function while maintaining an underlying lattice on which the polynomials are defined [18, 19]. An ensuing problem has been to determine the recurrence coefficients [18, 19].

In the case of the Rabi model, the recurrence coefficients are explicitly known [cf. Eqs. (17), (18)]. As we have seen in Sec. [II] the lattice is equidistant only in the special limiting case $\Delta=0$. Then the polynomials are proportional to the Charlier polynomials, which are related to each other according to (cf. Eq. (VI-1.7) of Ref. [8])

$$
\Delta_{+} C_{n}^{\left(\kappa^{2}\right)}(x)=n C_{n-1}^{\left(\kappa^{2}\right)}(x),
$$

where $\Delta_{+} u(x)=u(x+1)-u(x)$ denotes the forward finite difference operator 9]. Their weight function (21) satisfies a special form of the Pearson difference equation

$$
\Delta_{+} u(x)=\frac{\kappa^{2}-x-1}{x+1} u(x) .
$$

A non-zero value of $\Delta$ induces a norm-preserving deformation of the Charlier polynomials to non-classical discrete orthogonal polynomials and, at the same time, a nonuniform deformation of the underlying equidistant lattice. The spectrum of the Rabi model is then nothing but the discrete nonuniform lattice. The so-called $q$-analogs of the Charlier polynomials on nonuniform lattices with the lattice points $x(n)=\exp (2 w n)$ and $x(n)=\sinh (2 w n)$, respectively, with $w>0$ being some parameter, have been discussed in Sec. 3.6 of Ref. [9]. Yet those extensions are still classical orthogonal polynomials that are distinguished by the fact that all their properties are unambiguously determined by the secondorder difference equation of hypergeometric type which they satisfy [9]. Contrary to the main line of research of the discrete orthogonal polynomials community [18, 19], the problems here are (i) to characterize the class of nonclassical norm-preserving extensions of the classical discrete Charlier polynomials which encompasses the polynomials presented here (e.g. in terms of a suitable second order difference equation), (ii) to find generalizations of Eqs. (41) and (42) for $\Delta \neq 0$, and (iii) to determine the nonuniform lattice on which the polynomials are defined. The problems are typically intertwined, because the for- ward finite difference operator is expected to operate on $\Lambda$ (cf. Sec. 3 of Ref. 9]), whereas a second order difference equation arises on combining a relation of the type (41) with the defining three-term recurrence (cf. Sec. VI-1 of Ref. [8]). The required extension has to be such that the average density of lattice points is substantially preserved [note that Eq. (26) only marginally depends on $\Delta]$.

\section{CONCLUSIONS}

The spectrum of the Rabi model was shown to coincide with the support of the discrete Stieltjes integral measure in the orthogonality relations of recently introduced non-classical discrete orthogonal polynomials. This finding brings about a novel method of solving the Rabi, and similar to it, models. In the case of the Rabi model the method resulted in an analytic solution that is considerably simpler than Braak's solution [17, 23]. The eigenfunctions can be determined in terms of orthogonal polynomials, whereas the eigenvalues are found as the polynomial zeros. Thus any omission of an energy level could easily be identified. The simplicity of our analytic solution was rewarded by the fact that the number of ca. 1350 calculable energy levels per parity subspace in double precision obtained by a simple stepping algorithm is almost two orders of magnitude higher than is possible to obtain by means of Braak's solution [17, 23]. A valuable insight as to whether a model is integrable or chaotic is provided by the energy level statistics. Our results suggest that energy eigenvalues are rather closely distributed around a straight line 22].

Although we can rigorously prove our results only for $\kappa<1$, numerics and exactly solvable example suggest that the main conclusions are valid also for $\kappa \geq 1$. Our results could thus provide a reliable point of departure for the calculation of the dynamics of the Rabi model and its long-time evolution for all values of the dimensionless coupling $\kappa$. The latter could be important to a great variety of physical systems, including cavity and circuit quantum electrodynamics, quantum dots, polaronic physics and trapped ions [33 36]. With new experiments rapidly approaching the limit of the deep strong coupling regime $\kappa \gtrsim 1$, one expect such systems to open up a rich vein of research on truly quantum effects with implications for quantum information science and fundamental quantum optics [20, 33].

\section{ACKNOWLEDGMENT}

Continuous support of MAKM is largely acknowledged. 
[3] A. Moroz, Europhys. Lett. 100, 60010 (2012).

[4] Y.-Z. Zhang, arXiv:1304.3979 [quant-ph].

[5] A. Moroz, to appear in Ann. Phys. (N.Y.) (arXiv:1302.2565).

[6] I. I. Rabi, Phys. Rev. 49, 324 (1936).

[7] W. Gautschi, SIAM Review 9, 24 (1967).

[8] T. S. Chihara, An Introduction to Orthogonal Polynomials (Gordon and Breach, New York, 1978).

[9] A. F. Nikiforov, S. K. Suslov, V. B. Uvarov, Classical Orthogonal Polynomials of a Discrete Variable (Springer, Berlin, 1991).

[10] W. Koepf and D. Schmersau, J. Comput. Appl. Math. 90, 57 (1998).

[11] A. V. Turbiner and A. G. Ushveridze, Phys. Lett. A 126, 181 (1987).

[12] A. V. Turbiner, Commun. Math. Phys. 118, 467 (1988).

[13] C. M. Bender and G. V. Dunne, J. Math. Phys. 37, 6 (1996).

[14] A. Krajewska, A. Ushveridze, and Z. Walczak, hep-th/9601088

[15] R. Koc, M. Koca, and H. Tütüncüler, J. Phys. A: Math. Gen. 35, 9425 (2002).

[16] R. L. Fulton and M. Gouterman, J. Chem. Phys. 35, 1059 (1961).

[17] D. Braak, Phys. Rev. Lett. 107, 100401 (2011).

[18] L. Boelen, G. Filipuk, and W. Van Assche, J. Phys. A: Math. Theor. 44, 035202 (2011).
[19] P. A. Clarkson, J. Phys. A: Math. Theor. 46, 185205 (2013).

[20] J. Casanova, G. Romero, I. Lizuain, J. J. García-Ripoll, and E. Solano, Phys. Rev. Lett. 105, 263603 (2010).

[21] C. V. L. Charlier, Ark. Mat. Astr. Fys. 2, 1 (1905-6).

[22] M. Kus, Phys. Rev. Lett. 54, 1343 (1985).

[23] D. Braak, Ann. Phys. (Leipzig) 525, L23 (2013).

[24] The source code can be freely downloaded from http://www . wave-scattering. com/rabi.html.

[25] A. Moroz, arXiv:1205.3139 [quant-ph].

[26] K. Ziegler, J. Phys. A: Math. Theor. 45, 452001 (2012).

[27] Data files *gp2dp4n300.dat obtained for $(\kappa, \Delta)=$ $(0.2,0.4)$ and $n=300$ are available from http://www. wave-scattering.com/rabi.html.

[28] B. R. Judd, J. Phys. C: Solid State Phys. 12, 1685 (1979).

[29] M. Kus, J. Math. Phys. 26, 2792 (1985).

[30] Y.-Z. Zhang, J. Phys. A: Math. Theor. 45, 065206 (2012).

[31] J.-S. Caux and J. Mossel, J. Stat. Mech. P02023 (2011).

[32] E. T. Jaynes and F. W. Cummings, Proc. IEEE 51, 89 (1963).

[33] G. Khitrova, H. M. Gibbs, M. Kira, S. W. Koch, and A. Scherer, Nat. Phys. 2, 81 (2006).

[34] J. Bourassa, J. M. Gambetta, A. A. Abdumalikov, Jr., O. Astafiev, Y. Nakamura, and A. Blais, Phys. Rev. A 80, 032109 (2009).

[35] P. Forn-Díaz, J. Lisenfeld, D. Marcos, J. J. García-Ripoll, E. Solano, C. J. P. M. Harmans, and J. E. Mooij, Phys. Rev. Lett. 105, 237001 (2010).

[36] T. Niemczyk, et al, Nat. Phys. 6, 772 (2010). 